\title{
2D TO 3D RECTANGULAR WAVEGUIDE FILTER DESIGNS FROM LINEAR ITERATED PREDICTION SPACE MAPPING OPTIMIZATION
}

\author{
J. Hinojosa ${ }^{1}$, F. D. Quesada Pereira ${ }^{2}$ and A. Alvarez-Melcon ${ }^{2}$ \\ ${ }^{1}$ Departamento de Electrónica, Tecnología de Computadoras y Proyectos \\ ${ }^{2}$ Departamento de Tecnologías de la Información y las Comunicaciones \\ Universidad Politécnica de Cartagena \\ Plaza del Hospital nº 1 \\ 30202 Cartagena (Murcia) -SPAIN
}

Corresponding Author: Dr. Juan Hinojosa, Email: juan.hinojosa@upct.es, Fax: +34 968326400

\begin{abstract}
In this paper, an optimization procedure is described to align electromagnetic (EM) three-dimensional (3D) models with two-dimensional (2D) models for the design of $R F /$ microwave circuits. The optimization procedure is realized from a modified standard space mapping (SM) approach. The mapping function between the $2 \mathrm{D}$ and $3 \mathrm{D}$ parameter spaces is directly obtained from a linear iterated prediction method, which reduces the computational cost and also avoids inverse transformations. The linear iterated prediction $2 \mathrm{D}$ to 3D SM optimization of evanescent rectangular waveguide bandpass filters with inductive posts for the $2 \mathrm{D}$ models and non-inductive posts for the $3 \mathrm{D}$ models illustrate the advantages and the challenges of this approach. The proposed method is simple to be implemented, it requires a reduced computational cost and it can be useful for CAD environment with $2 \mathrm{D}$ and $3 D$ circuit structure electromagnetic (EM) analysis.
\end{abstract}


Key words: $C A D$; optimization techniques; microwave design; prediction methods; space mapping 


\section{INTRODUCTION}

Nowadays, the exploitation of electromagnetic (EM) simulators is one of the basic prerequisites for a successful activity in the development of accurate designs of microwave circuits. Moreover, innovative designs may be achieved using three-dimensional (3D) fullwave EM simulators. However, 3D EM simulators will not realize their full potential to the designer unless they are combined with optimization algorithms to automatically adjust designable parameters [1]. In a panel discussion on the state of microwave computer-aided design (CAD) in the year 2010, M. Mongiardo predicted much of the emphasis will shift to an optimization and design environment that allows integrated design [2]. In this sense, we want to focus this work in a particular case of design process, which is shown in the flowchart of Figure 1. The objective is to align the results of 2D and 3D EM simulators by means of an optimization procedure in order to avoid direct optimization of computationally intensive 3D models. In this way, the efficiency can be increased by employing a computationally efficient 2D EM simulator, together with a fast and reliable 2D to 3D iterative mapping technique.

The interest in interfacing various rigorous EM simulators into CAD frameworks, including the use of mapping between design spaces of different dimensionality, has been the purpose of numerous works [2-7]. To successfully interface EM simulators we need a powerful computational optimization tool to adjust designable parameters in order to meet design specifications. The two main classes are optimization techniques that require an initial solution such as a gradient-type approach [8], and techniques that do not require an initial solution such as genetic algorithm [9]. The use of these optimization techniques with rigorous EM simulators provides accurate designs. However, these approaches are very computationally intensive [10]. These last years, space mapping (SM) has been widely used in the optimization of microwave circuits integrating EM simulations with the purpose of reducing the computation time of the EM-based design process [7]. The SM approach 
establishes a mathematical link (mapping) between the spaces of the design parameters of two models: fine model (FM) and coarse model (CM). The FM is accurate and slow in computation time, whereas the CM is fast but less accurate. The use of the SM technique in an optimization tool consists in directing the bulk of CPU intensive evaluations to the CM, the FM being used only a few times during the design process.

In this paper, we propose a 2D to 3D SM approach combined with a linear iterated prediction procedure [11] in order to reduce the CPU time of the design process (Figure 1). This approach predicts the next 3D simulation tool design parameter space from the faster 2D simulation tool, until the required convergence between the 2D and 3D simulator responses is obtained. This linear iterated prediction 2D to 3D SM optimization procedure is a modified standard SM optimization approach [12]. The standard SM approach produces a mapping function $P$ from 3D design parameter space of the FM to 2D design parameter space of the CM for fixed continuous design specifications, of which the values are known at all frequency points. The application of the standard SM approach requires $m$ initial base points and, therefore, $m$ evaluations of computationally intensive 3D simulators. Moreover, it needs an inverse transformation of the mapping function $P$ generated between the 2D and 3D parameter spaces to obtain corresponding 3D model design, which makes difficult the optimization procedure when $P$ is not invertible. Our proposed technique is simpler and reduces the computational cost. It realizes the inverse mapping function $P$ from a linear iterated prediction 2D to 3D SM optimization procedure for fixed discrete design specifications. In this way, no generation of $m$ base points around continuous target response at the initial mapping and no inverse transformation of the mapping function are necessary. This paper is organized as follows. Section 2 presents the 2D to 3D SM concept, the formulation of the linear iterated prediction 2D to 3D SM optimization procedure, and the algorithm interfacing two EM simulators (2D and 3D) with the linear iterated prediction SM 
optimization for 2D to 3D microwave circuit designs. The resulting CAD framework is used in Section 3 to design C-band evanescent rectangular waveguide bandpass filters with noninductive dielectric posts, which present a great interest in satellite applications. The relevant information for the design of filters containing non-inductive dielectric posts is taken from the design of simpler filters based on inductive dielectric posts, together with the mapping technique developed in this paper. Finally, conclusions are presented in Section 4.

\section{2D TO 3D SPACE MAPPING OPTIMIZATION}

This Section describes the 2D to 3D SM concept [7], the formulation of the 2D to 3D SM optimization procedure based on a linear iterated prediction method [12], and the algorithm interfacing two EM simulators (2D and 3D) with this optimization approach for 2D to 3D microwave circuit designs.

2.1. $2 D$ to $3 D$ Space Mapping Concept. As in other SM-based optimization [7], two models are available for the device to be designed: a fine model (FM) and a coarse model (CM). Both CM and FM provide accurate EM analysis. The FM is very accurate to analyze arbitrary 3D geometries but computationally expensive. The CM is computationally efficient in its treatment of a variety of $2 \mathrm{D}$ geometries. In this way, the $\mathrm{CM}$ can be intensively evaluated without significant computational cost. The key idea is to extract the response of the real 3D structure, from a number of responses of a related 2D structure, which are faster to obtain.

The 2D to 3D SM approach (Figure 2) consists in finding an appropriate linear mapping $P$ from the design parameter space of a computationally efficient 2D EM simulator (CM) to the design parameter space of a computationally intensive 3D EM simulator (FM) for fixed discrete design specifications: 


$$
X_{3 \mathrm{D}}=P\left(X_{2 \mathrm{D}}\right)
$$

such that

$$
\left\|R_{2 D}\left(X_{2 \mathrm{D}}, f\right)-R_{3 \mathrm{D}}\left(P\left(X_{2 \mathrm{D}}, f\right)\right)\right\| \leq \varepsilon
$$

where

$$
\begin{gathered}
X_{2 \mathrm{D}}=\left(x_{1}, \ldots, x_{n}, y_{1} \ldots, y_{p}\right) \in \mathfrak{R}^{n+p} \\
X_{3 \mathrm{D}}=\left(x_{1}^{*}, \ldots, x_{n}^{*}, y_{1}^{*}, \ldots, y_{p}^{*}, z_{1}, \ldots, z_{k}\right) \in \mathfrak{R}^{n+p+k}
\end{gathered}
$$

are, respectively, optimal 2D and 3D design parameter spaces for a predefined value over the error criterion (2) and fixed design specifications. $R_{2 \mathrm{D}}$ and $R_{3 \mathrm{D}}$ are the corresponding $2 \mathrm{D}$ and 3D simulator responses and $f$ the frequency. $P \in \mathfrak{R}^{n+p}$ and the variables $z_{k}$ of an additional third dimension are known or fixed by the constraints, like for example the cut-off frequency band for the rectangular waveguides, the thickness of the substrate (planar technology), the height of dielectric posts, or by the designer.

Contrary to the standard SM concept [11], our approach (Figure 3) uses the design parameter space of the CM to produce a mapping function $P$ on the design parameter space of the FM in order that the response of the FM is in good agreement with the fixed discrete design specifications. Applied to the 2D to 3D SM approach, the mapping function $P$ is found by means of a design process that reveals the 3D design parameter space of the 3D simulator, 
$X_{3 D}=P\left(X_{2 D}\right)$, in such a way that the $3 \mathrm{D}$ simulator response (FM), $R_{3 D}\left(P\left(X_{2 D}, f\right)\right)$, is close enough to the target response, which is given by the optimal $2 \mathrm{D}$ simulator response (CM), $R_{2 D}\left(X_{2 D}, f\right)$, for the fixed discrete design specifications. The discrete design specifications are referred to those values defined between a frequency range and/or a frequency point. On the other hand, we define for continuous design specifications to those values known at all frequency points. In most practical cases of 3D structure design, it is difficult that the optimized responses of different dimensionality simulators coincide at all frequency points. They only agree in determined discrete design specifications, and therefore, the mapping function must be produced for these ones. Thus, to keep a reduced amount of FM simulations, the generation of $m$ base points around continuous target response at the initial mapping is omitted by contrast to the standard SM concept. The proposed 2D to 3D SM approach consists of two sub-processes: 1$)$ find a target response $R_{2 D}\left(X_{2 D}, f\right)$ according to the design specifications by optimizing the CM, which is the 2D simulator; 2) find $P$ and $X_{3 D}$ of the FM (3D simulator) by means of linear iterated prediction 2D to 3D $\mathrm{SM}$ optimization procedure, which is described in the next sub-section.

2.2. Linear Iterated Prediction $2 D$ to $3 D$ SM Optimization Procedure. The aim of this approach is to generate linear prediction models of the mapping function $P$ from $2 \mathrm{D}$ to $3 \mathrm{D}$ design parameter spaces by means of an iterated prediction procedure until the predicted values of the optimal 3D design parameters, $X_{3 \mathrm{D}}^{*}$, satisfy an error criterion (2) between the 2D and 3D simulator responses for fixed design specifications.

The linear prediction model used in the iterated prediction method for each variable $x$ ' in the 2D design parameter space to be mapped $x^{*}$ in the 3D design parameter space, has the form: 


$$
\begin{aligned}
& F: \mathfrak{R} \rightarrow \mathfrak{R} \\
& F_{x^{\prime}}\left(x^{\prime}\right)=x^{*}=a_{0 x^{\prime}}+a_{1 x^{\prime}} x^{\prime}
\end{aligned}
$$

The coefficients $\left(a_{0 x^{\prime}}, a_{1 x^{\prime}}\right)$ are iteratively calculated over $x^{\prime}$ by using two data sets of inputoutput pairs from the ith and $i$-1th iterations, respectively. Assume that the ith iteration has a data set $\left(X_{2 \mathrm{D}, i}^{\prime}, F\left(X_{2 \mathrm{D}, i}^{\prime}\right)\right)$ of the form:

$$
\begin{gathered}
X_{2 \mathrm{D}, i}^{\prime}=\left(x_{1, i}^{\prime}, \ldots, x_{n, i}^{\prime}, y_{1, i}^{\prime}, \ldots, y_{p, i}^{\prime}\right) \\
F\left(X_{2 \mathrm{D}, i}^{\prime}\right)=X_{3 D, i}^{*}=\left(x_{1, i}^{*}, \ldots, x_{n, i}^{*}, y_{1, i}^{*}, \ldots, y_{p, i}^{*}\right)
\end{gathered}
$$

where the inputs and outputs are the data defined in (6) and (7), respectively. In the same manner, we define $\left(X_{2 \mathrm{D}, i-1}^{\prime}, F\left(X_{2 \mathrm{D}, i-1}^{\prime}\right)\right)$ for the $i$-1th iteration. $X_{2 \mathrm{D}, i}^{\prime}$ and $X_{3 \mathrm{D}, i}^{*}$ are the predicted values in the 2D and 3D design parameter spaces for the ith iteration, respectively.

On the other hand, the next 3D prediction space model $P^{i}\left(X_{2 \mathrm{D}}\right)$ for iterated 3D design parameter space prediction has the form:

$$
\begin{aligned}
P^{i}: \mathfrak{R}^{n+p} & \rightarrow \mathfrak{R}^{n+p} \\
P^{i}\left(X_{2 \mathrm{D}}\right)= & \left(F_{X_{1}}\left(x_{1}\right), \ldots, F_{X_{n}}\left(x_{n}\right), F_{y_{1}}\left(y_{1}\right), \ldots, F_{y_{p}}\left(y_{p}\right)\right) \\
& =X_{3 \mathrm{D}, i+1}^{*}
\end{aligned}
$$


where $F$ are the linear prediction models (5) obtained from the ith and $i$-1th iterations for all the variables to be optimized. $X_{2 \mathrm{D}}$ corresponds to the optimal 2D design parameter space (3) and $X_{3 \mathrm{D}, i+1}^{*}=\left(x_{1, i+1}^{*}, \ldots, x_{n, i+1}^{*}, y_{1, i+1}^{*}, \ldots, y_{p, i+1}^{*}\right)$ (the variables $z_{k}$ are known or fixed) are the predicted values of the optimal 3D design parameters to be determined in the $i+1$ th iteration. The iterated prediction is performed from the predicted values $X_{3 \mathrm{D}, i+1}^{*}$ to construct the next $2 \mathrm{D}$ values $X_{2 \mathrm{D}, i+1}^{\prime}$, which are used to predict the next $3 \mathrm{D}$ values $X_{3 \mathrm{D}, i+2}^{*}$ and so on until the error criterion (2) is satisfied for fixed design specifications, or the user-defined number of iterations is exceeded.

2.3. Algorithm Flow. The algorithm flow can be described from the following steps $\left(R_{2 \mathrm{D}}\right.$ and $R_{3 \mathrm{D}}$ are the corresponding $2 \mathrm{D}$ and $3 \mathrm{D}$ simulator responses):

1) Set $i=0$, optimize $R_{2 \mathrm{D}}$ for fixed design specifications using SM, and extract the value $X_{2 \mathrm{D}}$

2) Evaluate $R_{3 \mathrm{D}}$ with $X_{3 \mathrm{D}, 0}^{*}=X_{2 \mathrm{D}}$;

3) Optimize $R_{2 \mathrm{D}}$ using SM to find previous $R_{3 \mathrm{D}}$ and extract the value $X_{2 \mathrm{D}, 0}^{\prime}$;

4) Obtain the slopes of (5) from $X_{3 \mathrm{D}, 0}^{*}$ and $X_{2 \mathrm{D}, 0}^{\prime}$;

5) Calculate $X_{3 \mathrm{D}, 1}^{*}$ using (8) and add $X \%$ over each variable of $X_{3 \mathrm{D}, 1}^{*}$, such that $X_{3 \mathrm{D}, 1}^{*}=X_{3 \mathrm{D}, 1}^{*}+X_{3 \mathrm{D}, 1}^{*} \times X \%$;

6) Evaluate $R_{3 \mathrm{D}}$ with $X_{3 \mathrm{D}, 1}^{*}$;

7) Set $i=i+1$, optimize $R_{2 \mathrm{D}}$ using SM to find previous $R_{3 \mathrm{D}}$ and extract the values $X_{2 \mathrm{D}, i}^{\prime}$ 
8) Obtain the coefficients of the linear prediction models (5) from the two last iterations;

9) Calculate $X_{3 \mathrm{D}, i+1}^{*}$ using (8);

10) Evaluate $R_{3 \mathrm{D}}$ with $X_{3 \mathrm{D}, i+1}^{*}$;

11) Compare $R_{2 \mathrm{D}}$ of Step 1 with $R_{3 \mathrm{D}}$ of Step 10; if the error criterion (2) is not satisfied for fixed design specifications and if the user-defined number of iterations is not exceeded go to Step 7; else END.

The SM used in the Steps 1, 3 and 7 may be of the same or different type. $X \%$ in the Step 5 allows increasing the convergence rate of this iterative optimization method.

\section{RESULTS}

The linear iterated prediction 2D to 3D SM optimization technique described in Section 2 was applied to first-order and second-order evanescent rectangular waveguide bandpass filters with dielectric posts. The dielectric posts in 2D are inductive, that is the height of the posts is the same as the internal height of the rectangular waveguide. In this case, the EM filter analysis can be made simpler. In 3D (Figures 4 and 5), the height of the posts is lower than the internal height of the rectangular waveguide and, therefore, the structure is completely 3D. The posts are centered in the cavity of length $l_{C}$. Rectangular waveguide size is standard: WR-229 $(a=58.1 \mathrm{~mm}, b=29.05 \mathrm{~mm})$. Other parameters are: $c=31.1 \mathrm{~mm}, h_{1}=17.05 \mathrm{~mm}$ (the height of the posts in 3D as shown in Figures 4(b) and 5(b)) and $\varepsilon_{r}=4$ (dielectric posts). The 3D design parameters to be optimized are $X_{3 \mathrm{D}}=\left(l_{c}, \phi_{1}\right) \mathrm{mm}$ and $X_{3 \mathrm{D}}=\left(l_{c}, \phi_{1}, d_{c}\right)$ $\mathrm{mm}$ for the first-order and second-order filters, respectively (see Figures 4 and 5). 2D and 3D EM simulations were provided from a surface integral equation simulator [13] and a commercially available finite element simulator, respectively. The simulation times were, 
respectively, 3:14 minutes and 1:12 hours for the 2D and 3D simulators and for the secondorder evanescent rectangular waveguide bandpass filter (Figure 4) with 101 frequency points. In the case of the first-order filter, the design specifications are $f_{0}=4 \mathrm{GHz}$ (resonant frequency) and 3dB bandwidth of $160 \mathrm{MHz}$. The design specifications for the second-order filter are $f_{0}=4 \mathrm{GHz}$, ripple level in the passband of $12 \mathrm{~dB}$ and ripple bandwidth of $80 \mathrm{MHz}$. The error criterion (2) is $\varepsilon \leq 1 \mathrm{~dB}$ in the $3 \mathrm{~dB}$ bandwidth for both filters.

In the algorithm, the 2D optimizations (steps 1, 3 and 7) were obtained from the same type that the linear iterated prediction SM method. The optimal 2D design parameters (Step 1) verifying the above design specifications were $X_{2 \mathrm{D}}=\left(l_{c}, \phi_{1}\right)=(37,5.7) \mathrm{mm}$ and $X_{2 \mathrm{D}}=\left(l_{C}, \phi_{1}, d_{C}\right)=(92,5.7,55) \mathrm{mm}$ for the first-order and second-order evanescent rectangular waveguide bandpass filters, respectively. They constitute the target responses for the linear iterated prediction 2D to 3D SM optimization procedure. The responses of the surface integral equation and finite element simulators at these optimal 2D design parameters are shown in Figures 6 and 7 for the first-order and second-order filters, respectively. As it can be seen, great discrepancies are evident before 2D to 3D SM optimization.

Applying the above algorithm flow, we have successful obtained the optimal 3D design parameters for both filters. The solutions of these filters were achieved with five and four 3D simulator evaluations (included 3D simulator evaluation at starting point Step 2) for $X=0 \%$ and $X=10 \%$, respectively. The optimal 3D design parameters provided with the linear iterated prediction 2D to 3D SM optimization technique were $X_{3 \mathrm{D}}^{*}=(39.98,13.29) \mathrm{mm}$ and $X_{3 \mathrm{D}}^{*}=(98.8,13.18,58.67) \mathrm{mm}$. Figures 8 and 9 show the 2D and 3D simulator $\left|S_{i j}\right|$ responses corresponding, respectively, to the first-order and second-order evanescent rectangular waveguide bandpass filters with the optimal 2D and 3D design parameters. It can be seen the response accuracy for both filters is good according to the design specifications 
and the error criterion. By applying the proposed technique to several second-order structures, the coupling coefficients of higher order filters can be efficiently obtained for the design of more complex microwave filter [14].

\section{CONCLUSION}

A linear iterated prediction 2D to 3D SM optimization algorithm is presented. The key idea is to reduce the number of analysis needed with time consuming 3D electromagnetic simulators, by performing optimization with fast $2 \mathrm{D}$ simulators, and a proper mapping between the two spaces. The application of this technique to the optimization of $2 \mathrm{D}$ to $3 \mathrm{D}$ first-order and second-order evanescent rectangular waveguide bandpass filters with dielectric posts has needed only a few iterations until the required convergence is reached, therefore leading to a reduced computational cost. This procedure can compete against other "state of the art" optimization procedures. Moreover, it does not require complex mathematical knowledge and tools, and therefore, it can be easily integrated in CAD environments with 2D and 3D circuit structure EM analysis. Consequently, it can also be easily used by circuits and systems designers. 


\section{ACKNOWLEDGEMENTS}

This work was supported by Ministerio de Educación y Ciencia of Spain (TEC2007-67630C03-02/TCM).

\section{REFERENCES}

1. J. W. Bandler, R. M. Biernacki, S. H. Chen, L. W. Hendrick, and D. Omeragic, Electromagnetic optimization of 3-D structures, IEEE Trans Microwave Theory Tech 45 (1997), 770-779.

2. R. C. Booton, Microwave CAD in the year 2010 - A panel discussion, Int J of RF and Microwave CAE 9 (1999), 439-447.

3. F. Arndt, Th. Sieverding, T. Wolf, and U. Papziner, Optimization-oriented design using mode-matching simulators in common circuit CAD tools, Int J of RF and Microwave CAE 7 (1996), 37-51.

4. J. W. Bandler, R. M. Biernacki, S. H. Chen, and P. A. Grobelny, Optimization technology for nonlinear microwave circuits integrating electromagnetic simulations, Int J of RF and Microwave CAE 7 (1997), 6-28.

5. R. Levy, R. V. Snyder, and G. Matthaei, Design of microwave filters, IEEE Trans Microwave Theory Tech 50 (2002), 783-793.

6. D. Swanson, and G. Macchiarella, Microwave filter design by synthesis and optimization, IEEE Microwave Mag 8 (2007), 55-69. 
7. J. W. Bandler, Q. S. Cheng, S. A. Dakroury, A. S. Mohamed, M. H. Bakr, K. Madsen, and J. Sondergaard, Space mapping: The state of the Art, IEEE Trans Microwave Theory Tech 52 (2004), 337-361.

8. S. Rosloniec, Algorithms for computer-aided design of linear microwave circuit, Artech House, Norwood, MA, 1990.

9. Y. Rahmat-Samii, and E. Michielssen, Electromagnetic optimization by genetic algorithms, John Wiley \& Sons, 1999.

10. S. F. Peik, and Y. L. Chow, Genetic algorithms applied to microwave circuit optimization, Proc of Asia Pacific Microwave Conference (1997), 857-860.

11. J. W. Bandler, R. M. Biernacki, S. C. Chen, P. A. Grobelny and R. H. Hemmers, Space mapping technique for electromagnetic optimization, IEEE Trans Microwave Theory Tech 42 (1994), 2536-2543.

12. J. Doyne Farmer, and J. J. Sidorowich, Prediction chaotic time series, Phys Rev Lett 59 (1987), 845-848.

13. F. D. Quesada Pereira, V. E. Boria Esbert, J. Pascual García, A. Vidal Pantaleoni, A. Alvarez Melcón, J. L. Gomez Tornero, and B. Gimeno, Efficient analysis of arbitrary shaped inductive obstacles in rectangular waveguides using a surface integral-equation formulation, IEEE Trans Microwave Theory Tech 55 (2007), 715-721.

14. R. Levy, R. V. Snyder, and G. Matthaei, Design of microwave filters, IEEE Trans Microwave Theory Tech 50 (2002), 783-793. 
Figure captions:

Figure $1 \quad$ Flowchart of the design process

Figure 2 Illustration of the 2D to 3D SM concept

Figure $32 \mathrm{D}$ to 3D SM approach

Figure 4 First-order evanescent rectangular waveguide bandpass filter with one post. (a) Top view. (b) Cross-section view

Figure 5 Second-order evanescent rectangular waveguide bandpass filter with two posts.

(a) Top view. (b) Cross-section view

Figure 6 Magnitudes of $S_{11}$ and $S_{21}$ of the first-order evanescent rectangular waveguide bandpass filter (one post) before 2D to 3D SM optimization 2D simulator

$----3 \mathrm{D}$ simulator

Figure 7 Magnitudes of $S_{11}$ and $S_{21}$ of the second-order evanescent rectangular waveguide bandpass filter (two posts) before 2D to 3D SM optimization 2D simulator

$----3 \mathrm{D}$ simulator 
Figure 8 First-order evanescent rectangular waveguide bandpass filter with one post: 2D and 3D model responses for the optimal 2D and 3D design parameters

2D simulator

$----3 \mathrm{D}$ simulator

Design specifications

Figure 9 Second-order evanescent rectangular waveguide bandpass filter with two posts:

2D and 3D model responses for the optimal 2D and 3D design parameters

2D simulator

$----3 \mathrm{D}$ simulator

— Design specifications 
Figure 1

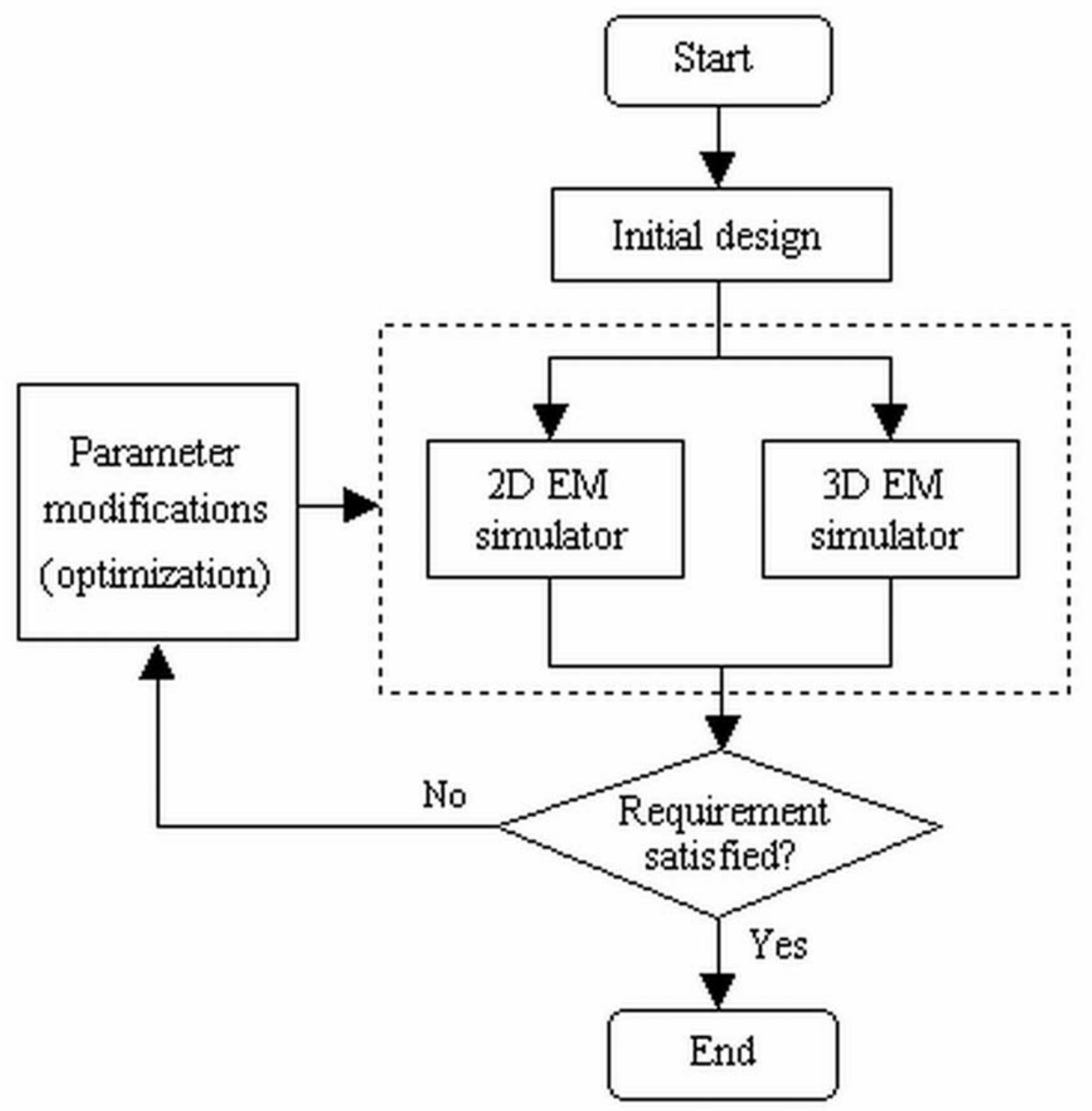


Figure 2

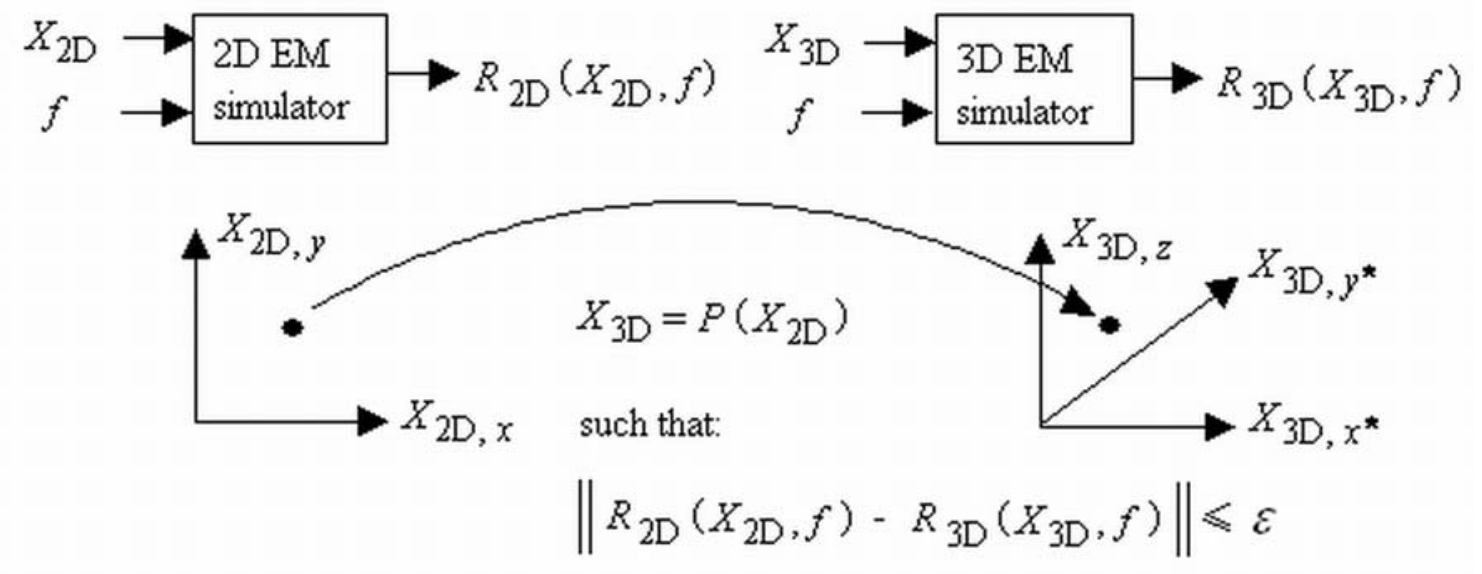


Figure 3

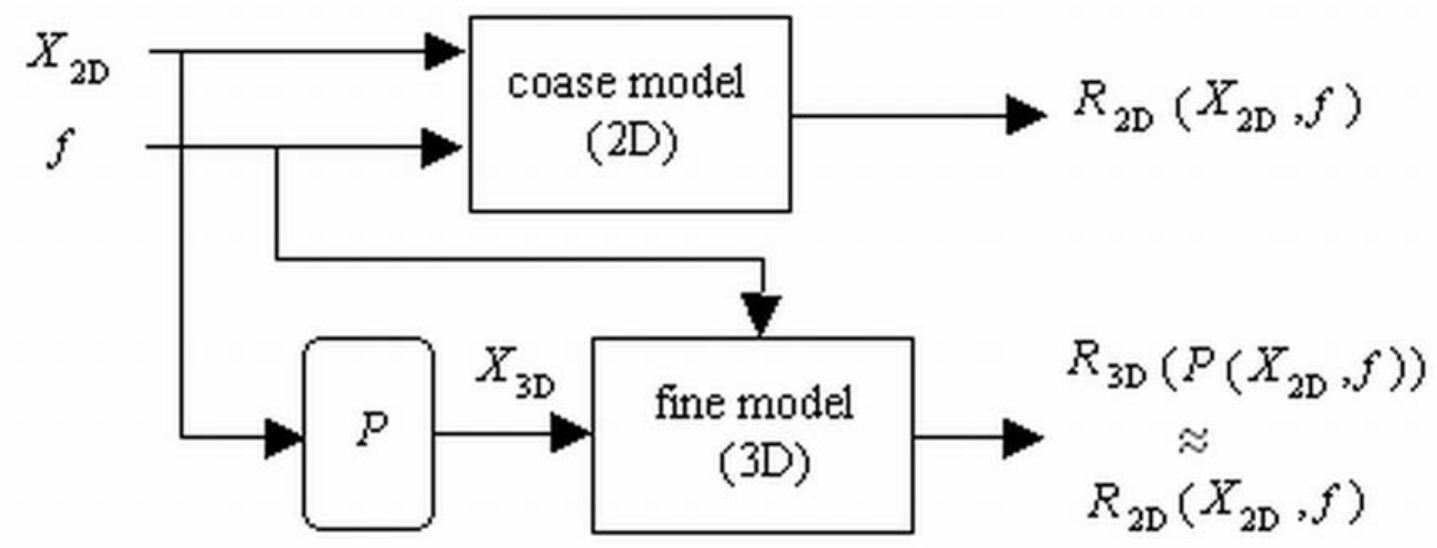


Figure 4

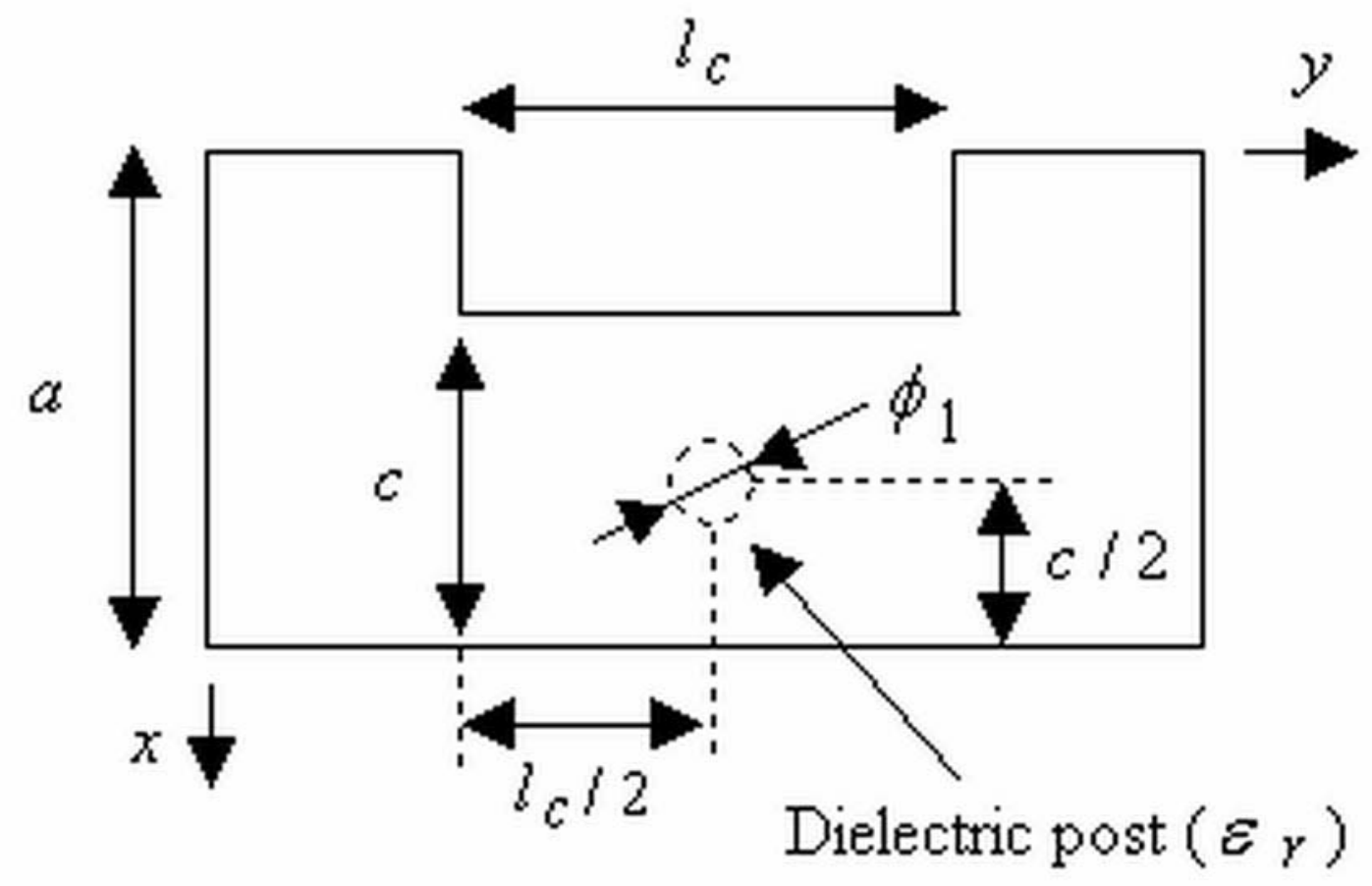

(a) 
Figure 4

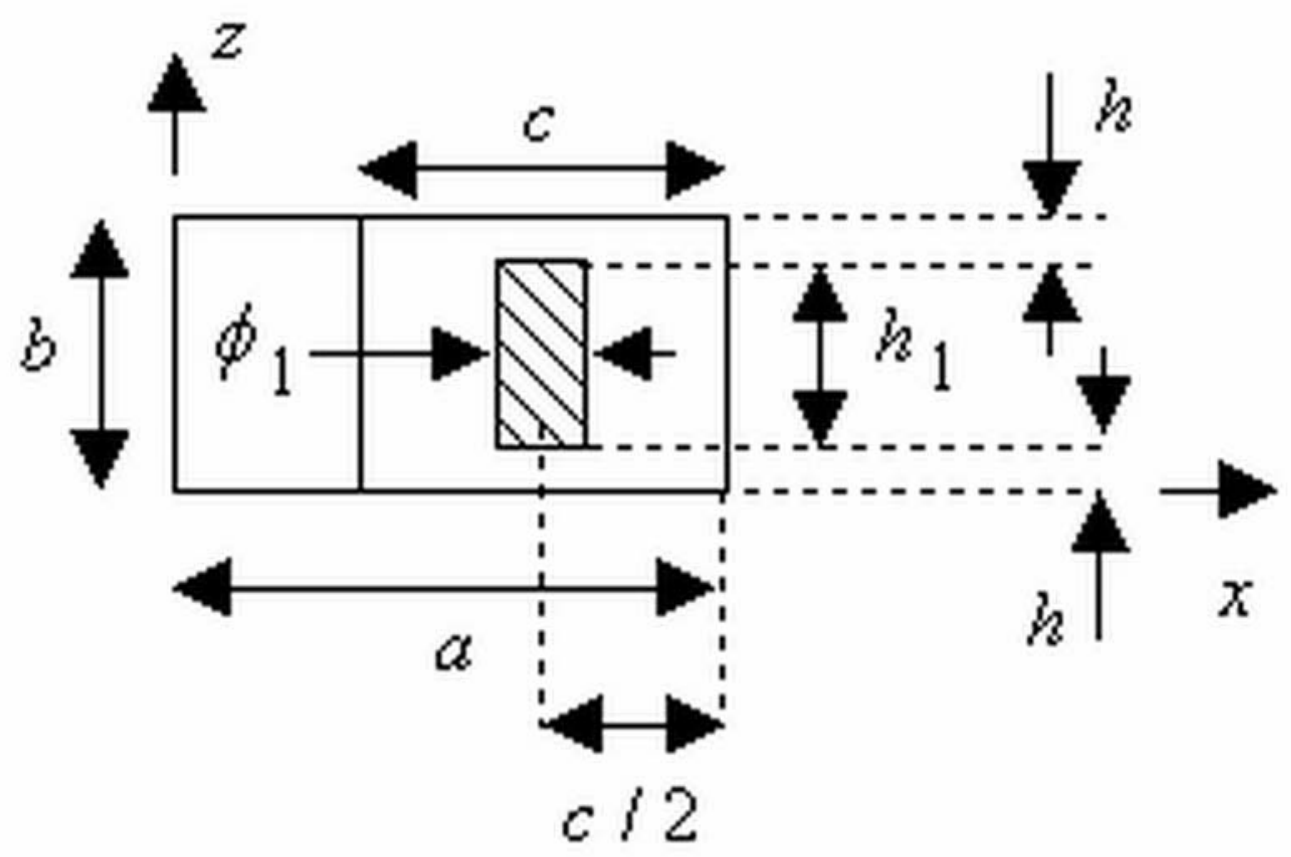

(b) 
Figure 5

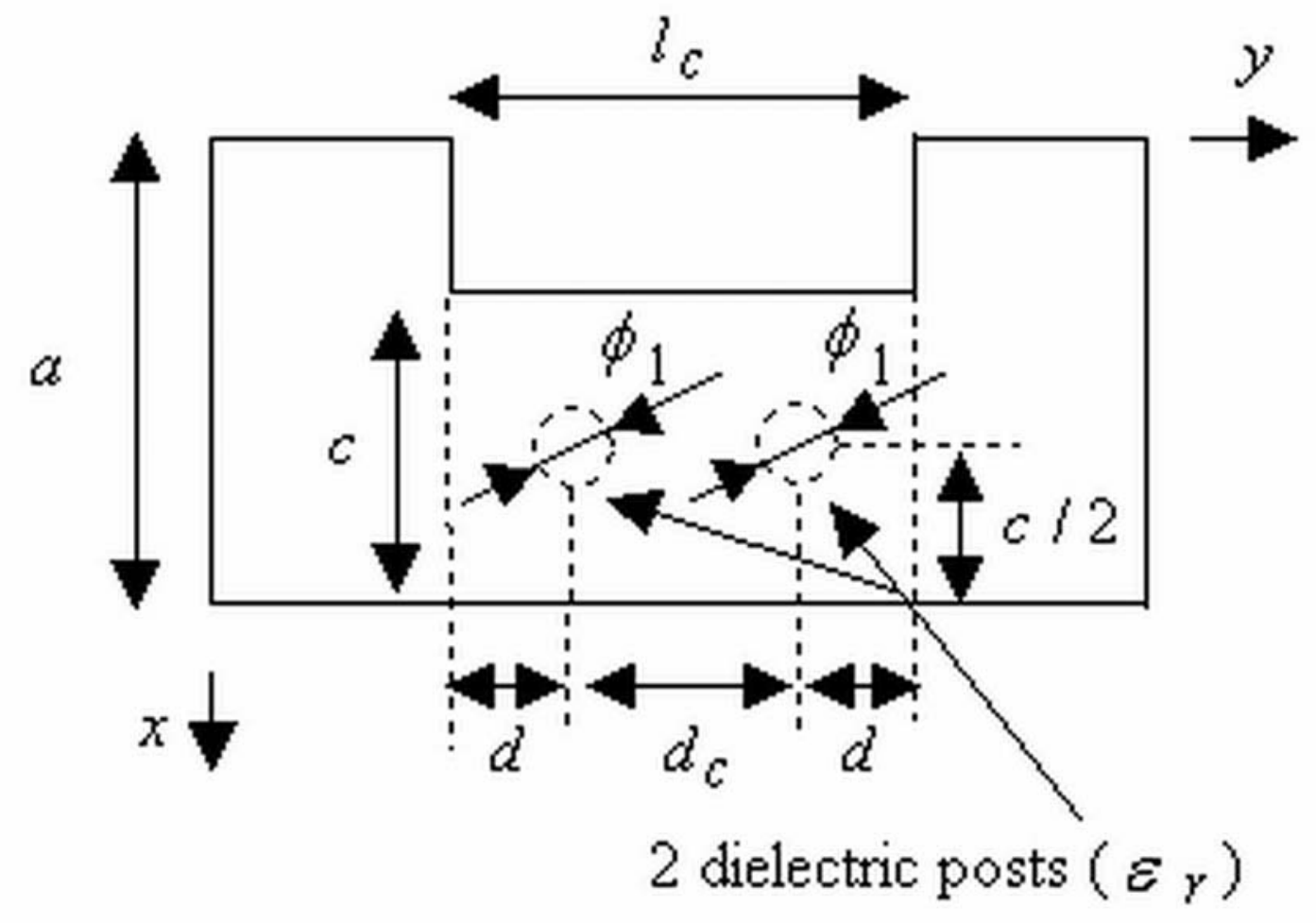

(a) 
Figure 5

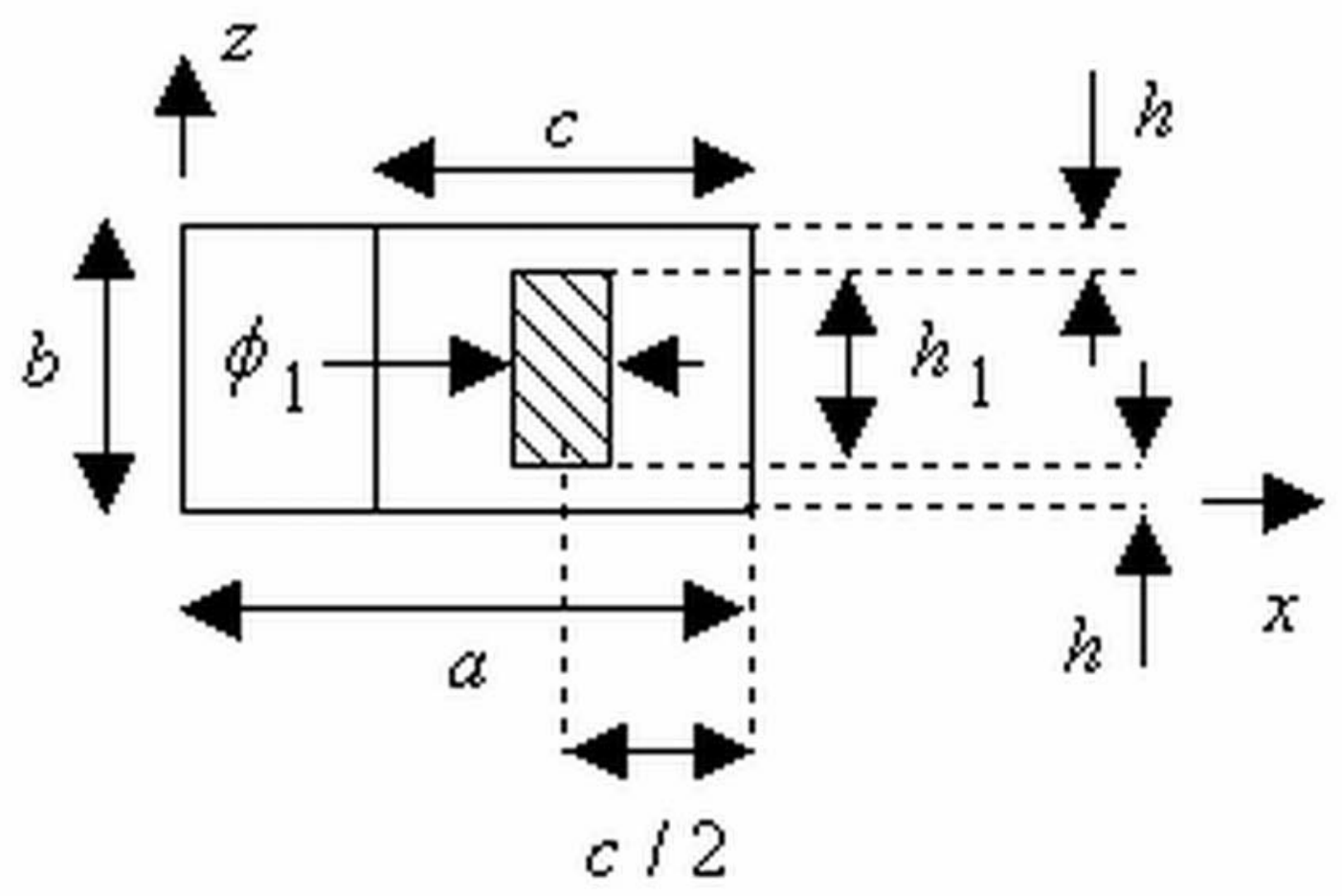

(b) 
Figure 6

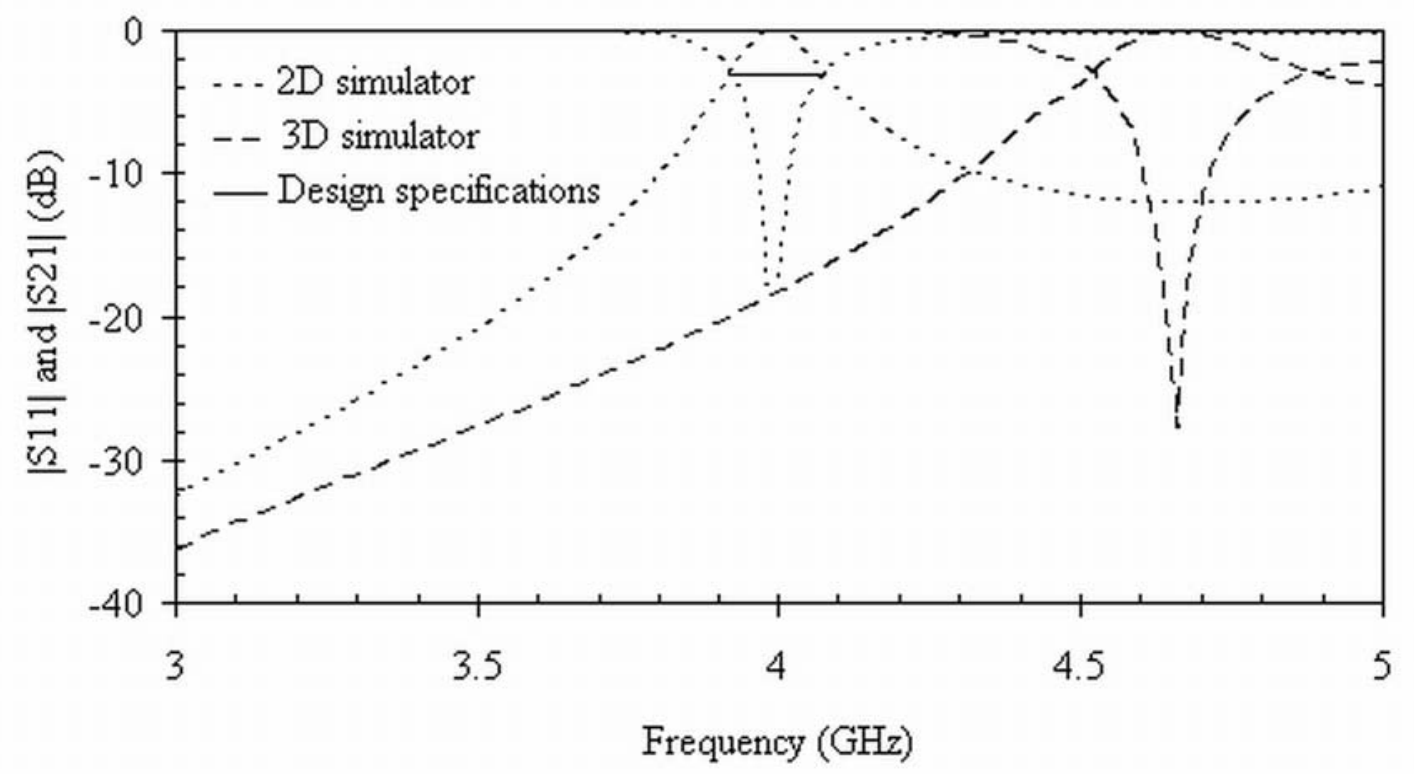


Figure 7

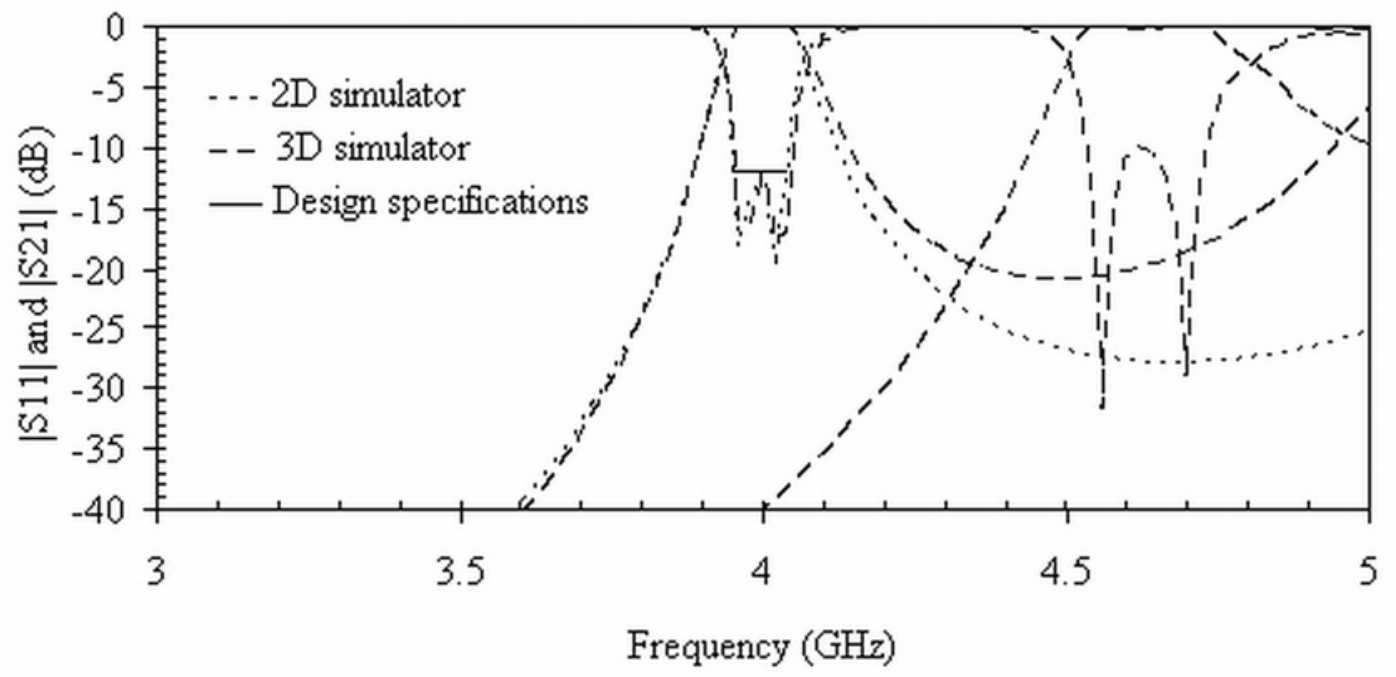


Figure 8

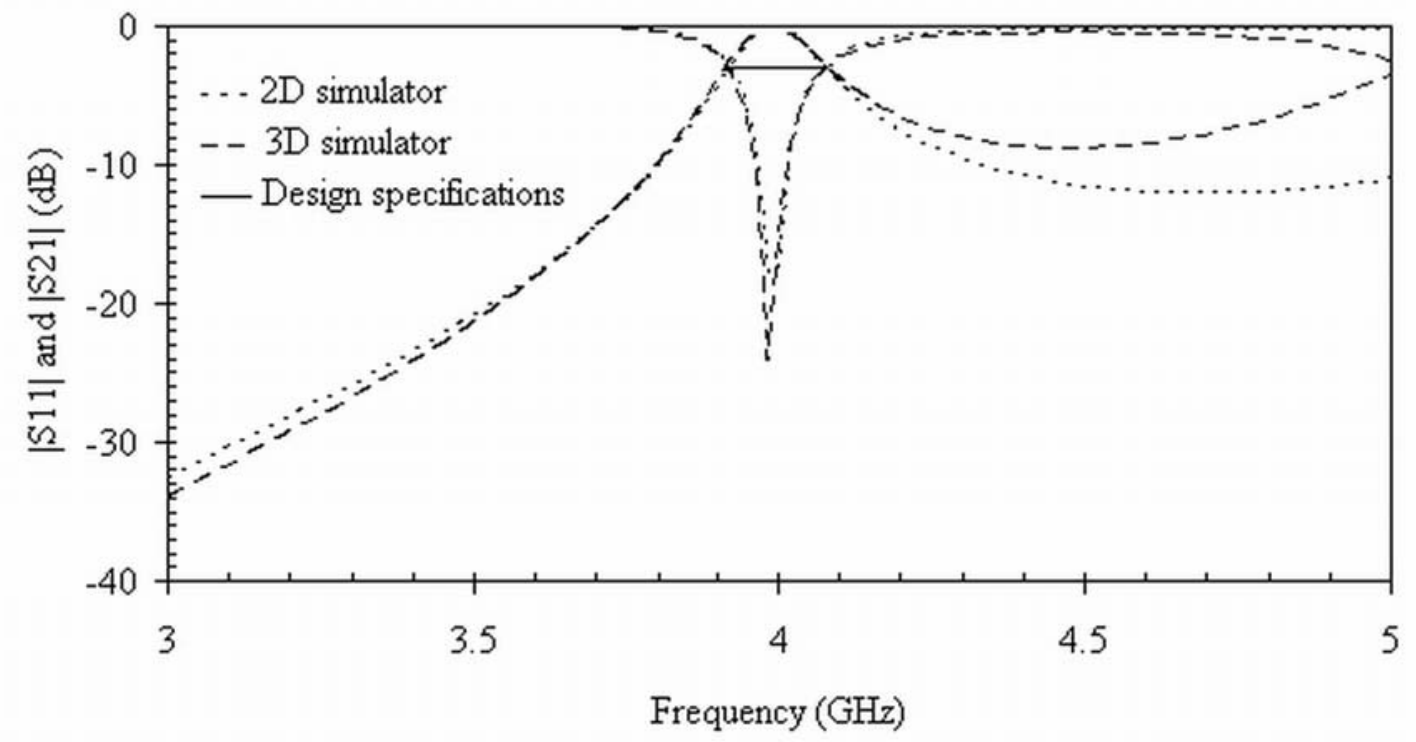


Figure 9

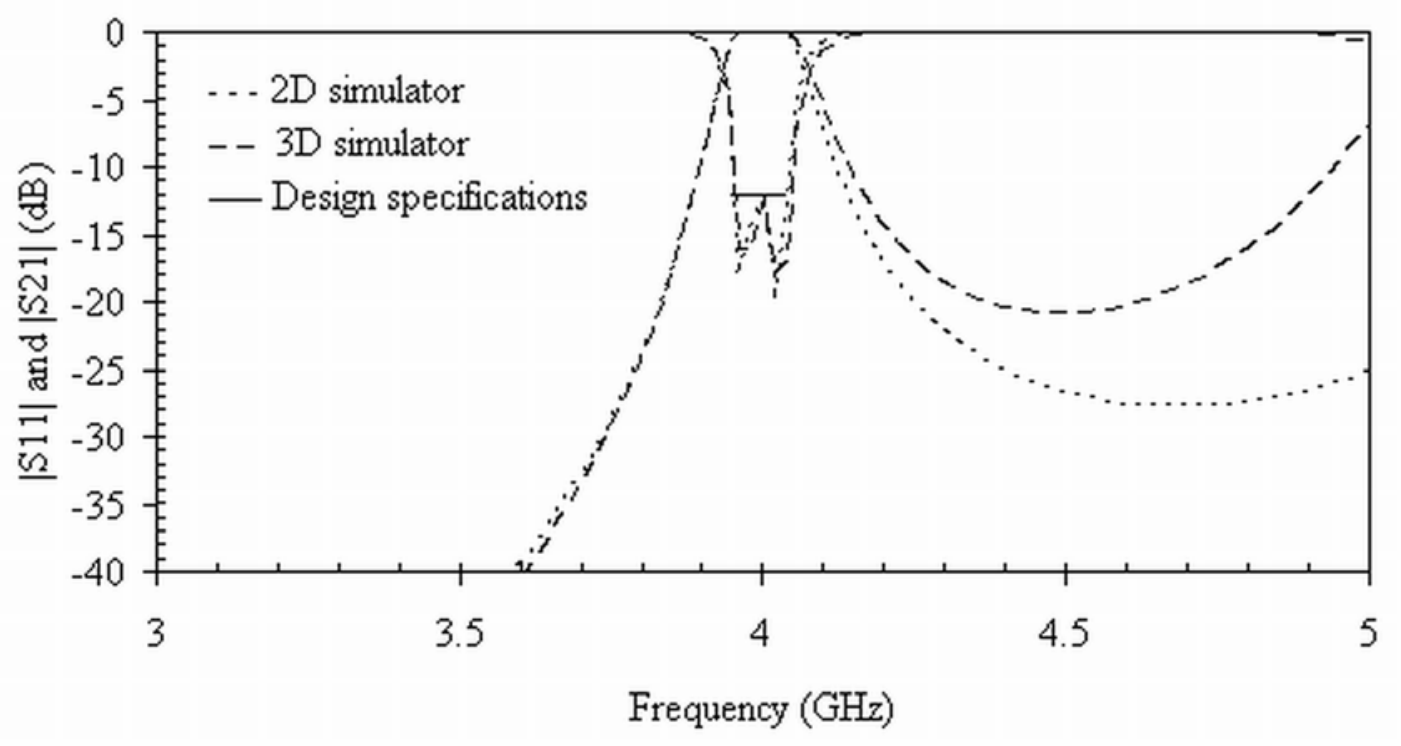

